\title{
Macrolides and pneumonia
}

\author{
Jeremy Stuart Brown, ${ }^{1}$ Adam T Hill ${ }^{2}$
}

Community acquired pneumonia (CAP) is increasing in incidence, and is presently responsible for about $1 \%$ of emergency medical admissions with an associated mortality of between $5 \%$ and $24 \%$. Improving the outcome of CAP is, therefore, a major health priority, but what is the best method to do this? Many audits have shown poor adherence to national guidelines for the management of CAP, with the inference that this may negatively affect outcome. One example of poor compliance is with empirical antibiotic therapy, the mainstay of treatment for most patients. Major guidelines for CAP advise that patients admitted to hospital with moderate and severe CAP should be treated with a combination of a $\beta$ lactam and a macrolide, ${ }^{1}$ yet large national audit datasets show that over a third of patients may be treated with $\beta$ lactams alone. ${ }^{2}{ }^{3}$ If the poor compliance with CAP guidelines affects outcome this would have serious implications for care of these patients and provide an obvious mechanism to improve their mortality. Conversely, using

\footnotetext{
${ }^{1}$ Centre for Inflammation and Tissue Repair, University College London, London, UK; ${ }^{2}$ Department of Respiratory Medicine, Royal Infirmary and University of Edinburgh, Edinburgh, UK
}

Correspondence to Professor Jeremy Stuart Brown, Centre for Inflammation and Tissue Repair, University College London, Rayne Institute, 5 University Street, London WC1E 6JJ, UK; jeremy.brown@ucl.ac.uk dual therapy when monotherapy is adequate will incur unnecessary additional cost, an increased risk of complications, and potentially encourage antibiotic resistance among commensal organisms.

The guidance to use dual $\beta$ lactam and macrolide antibiotics for empirical therapy of CAP for hospitalised patients moderate and severe CAP is based on microbiology data showing around 20\% of CAP is caused by 'atypical' organisms (Mycoplasma pneumoniae, Chlamydophila pneumoniae and Legionella pneumophila); these do not respond to $\beta$ lactams but are sensitive to macrolides. However, there are almost no controlled trial data on the relative efficacy of these regimens to support the guideline recommendations. In this context, Rodrigo et al have used data from the British Thoracic Society (BTS) national audits to retrospectively compare the outcome of CAP for 3239 patients treated with dual $\beta$ lactam and macrolide antibiotics, versus 2001 patients treated with $\beta$ lactams alone. ${ }^{3}$ The authors found that after adjusting for CURB65 scores, age, sex, comorbidities, intravenous administration of antibiotics, nursing home residency and admission to intensive care units (ICU), the OR of mortality of patients treated with dual therapy was 0.72 (CI 0.60 to 0.85 ) compared with those treated with $\beta$ lactam alone. Analysing the data according to CURB65 score indicated the beneficial effect of dual therapy was mainly for patients with moderate severity CAP (CURB65 2) who had a startling mortality OR of 0.54 (CI 0.41 to 0.72 ) compared with patients given $\beta$ lactams alone. There was also some effect for patients with severe CAP (CURB65 3+, OR 0.76 CI 0.60 to 0.96 ) but none for those with mild CAP (CURB65 $0-1$, OR 0.80 CI 0.56 to 1.16 ).

On the face of it, the Rodrigo et al data provide strong support for the guideline recommendation for dual $\beta$ lactam and macrolide therapy for CAP admitted to hospital. However, retrospective analysis of patient data has major intrinsic limitations. For instance, the results presented by Rodrigo et al will be affected by selection bias, and have to be interpreted with no data on the preadmission or duration of antibiotic use, use of other hospital therapies (eg, supplemental oxygen or thromboprophylaxis), accuracy of the records of antibiotic use, and information on the microbiological aetiology of the included patients. Furthermore, the quite high mortality for CAP patients in the BTS audit (24\%) compared with most prospective studies (around 6-14\%) ${ }^{1}$ perhaps indicates there is an overall selection bias in the patients included in this study, with possibly the inclusion of fewer patients with less severe CAP who are only admitted for short periods and could be more readily missed by the audit. This would reduce the general applicability of the results from the Rodrigo study. These shortcomings are partially overcome by the large numbers of patients included in the Rodrigo study and the use of multivariate analysis to adjust for the effects of known confounders, such as age and comorbidities. In addition, their study does not have to be taken in 
isolation; there are, in fact, several preceding cohort studies comparing outcomes for dual $\beta$ lactam and macrolide antibiotics versus single-agent $\beta$ lactam therapy. Some, but not all, of these studies are largely compatible with the Rodrigo data, showing improved outcomes for patients receiving dual therapy. ${ }^{4}$

However, despite this, selection bias and residual confounding of the results are still a major problem; in the Rodrigo study, patients receiving dual therapy were slightly younger, had fewer comorbidities, and were less likely to be admitted from a nursing home. ${ }^{3}$ Similarly, a propensity study found similar findings with dual therapy more common in younger patients with less comorbidity. ${ }^{6}$ In addition, the combination group was more likely to receive intravenous antibiotics and intensive care support. Hence, there may have been selection bias for patients with a better outcome to receive dual $\beta$ lactam and macrolide therapy, and residual confounding factors alone could explain the positive effects of dual therapy versus $\beta$ lactam. Furthermore, a contradictory finding in the Rodrigo study was lack of effects of dual therapy on the need for inotropic support, mechanical ventilation and ICU admission. Unless dual therapy can reduce the need for inotropes, mechanical ventilation or admission to ICU, it is difficult to work out how the addition of a macrolide improves survival. A comparison on the causes of death between these two groups would be helpful. Additional data that would be interesting and potentially supportive of a beneficial effect of dual $\beta$ lactam and macrolide therapy versus $\beta$ lactam alone would be length of stay, change in oxygen requirements, and rate of fall of inflammatory markers, such as $\mathrm{C}$ reactive protein.

Is it plausible that addition of a macrolide to a $\beta$ lactam could reduce mortality of patients with moderately severe CAP by approaching 50\%? That the effects are concentrated in patients with moderately severe CAP might be expected as these patients have a much higher mortality than mild disease, but might not be too sick for intervention to readily alter the clinical course. But as known 'atypical' organisms are present in only $20 \%$ of CAP cases in UK studies, an effect of dual therapy approaching 50\% is unlikely to be explained by treatment of these causes of CAP alone. Hence, there would have to be a benefit of macrolide therapy for patients not infected with one of the known 'atypical' organisms, and several alternative potential mechanisms can be hypothesised. Dual therapy with a macrolide and a $\beta$ lactam may be more effective at rapid control of bacterial numbers during infection with non-'atypical' organisms (mainly Streptococcus pneumoniae) than a single-agent $\beta$ lactam. Many cases of CAP are dual to mixed infection, usually $S$ pneumoniae, in combination with a respiratory virus, $M$ pneumoniae, or $C$ pneumoniae; it is possible that additional unknown macrolide-sensitive but $\beta$ lactam-insensitive organisms are present in many cases of CAP. Finally, CAP is often associated with a strong inflammatory response that contributes towards the development of consolidation, septic shock and acute lung injury. As a consequence, the immunomodulatory effects of macrolides that seem to be beneficial in airways infection could also have positive benefits for patients with pneumonia. Additional research in these areas would help identify whether any of these mechanisms are plausible, and therefore support the concept that dual therapy affects mortality in patients with CAP.

Overall, the data presented by Rodrigo et $\mathrm{al}$, and some of the previous retrospective cohort studies, support the existing guidelines by indicating that dual therapy with a $\beta$ lactam and macrolide is beneficial for patients with CAP. If this is confirmed, applying the guidelines to all patients admitted to hospital with CAP should have a significant effect on overall mortality due to CAP. However, these retrospective analyses are subject to bias, and in the absence of randomised controlled trials there can be no certainty that dual therapy is truly beneficial. CAP is common and clinically important, so it is surprising that we are still not able to answer the relatively simple question of whether the recommended empirical regimen is appropriate and has benefits over simpler therapeutic options. We believe that an adequately powered randomised controlled trial of dual therapy with a $\beta$ lactam and macrolide versus a $\beta$ lactam alone for patients with moderate or severe CAP admitted to hospital is a high priority, and may prove essential for future attempts to improve the mortality due to CAP. In the meantime, empirical antibiotics for CAP should follow national guideline advice.

Competing interests None.

Provenance and peer review Commissioned; internally peer reviewed.

To cite Brown JS, Hill AT. Thorax 2013;68:404-405.

Published Online First 5 March 2013

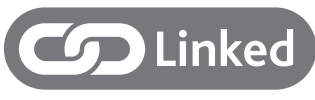

http://dx.doi.org/10.1136/thoraxjnl-2012-202296

Thorax 2013;68:404-405.

doi:10.1136/thoraxjnl-2012-202425

\section{REFERENCES}

1 Lim WS, Baudouin SV, George RC, et al. BTS guidelines for the management of community acquired pneumonia in adults: update 2009. Thorax 2009;64 (Suppl 3):iii1-55.

2 Lim WS, Woodhead M, British Thoracic Society. British Thoracic Society adult community acquired pneumonia audit 2009/10. Thorax 2011;66:548-9.

3 Rodrigo C, McKeever T, Woodhead M, et al. Single versus combination antibiotic therapy in adults hospitalised with community acquired pneumonia. Thorax 2013;68:493-5.

4 Tessmer A, Welte T, Martus $P$, et al. Impact of intravenous $\beta$-lactam/macrolide versus $\beta$-lactam monotherapy on mortality in hospitalized patients with community-acquired pneumonia. J Antimicrob Chemother 2009;63:1025-33.

5 Asadi L, Sligl WI, Eurich DT, et al. Macrolide-based regimens and mortality in hospitalized patients with community-acquired pneumonia: a systematic review and meta-analysis. Clin Infect Dis 2012;55:371-80.

6 Paul M, Nielsen AD, Gafter-Gvili A, et al. The need for macrolides in hospitalised community-acquired pneumonia: Propensity analysis. Eur Respir J 2007;30:525-31. 